\title{
Brown dwarfs and star forming regions in the framework of the Spanish Virtual Observatory
}

\author{
Enrique Solano $^{1}$, Eduardo L. Martín ${ }^{2}$ and José A. Caballero ${ }^{3}$ \\ ${ }^{1}$ INTA - Laboratorio de Astrofísica Espacial y Física Fundamental, \\ P.O. Box 50727, E-28080 Madrid, Spain \\ email: esm@laeff.inta.es \\ ${ }^{2}$ Instituto de Astrofísica de Canarias, \\ C/ Vía Láctea s/n, E-38200, La Laguna, Tenerife, Spain \\ email: ege@iac.es \\ ${ }^{2}$ Max-Planck-Institut für Astronomie, Königstuhl 17, D-69117 Heidelberg, Germany \\ email: caballero@mpia.de
}

In this paper we describe three lines of work that are being developed in the framework of the Spanish Virtual Observatory.

- Discovery of new ultra-cool brown dwarfs

Building a statistically significant census of substellar objects implies the use of queries that combine attributes available from different archives, an approach out of the scope of the 'classical' methodology but that perfectly fits into the Virtual Observatory. Using 2MASS, DENIS and SDSS and Aladin as VO-tool, we are focusing on the nearby $(d \leqslant 10 \mathrm{pc})$ population of brown dwarfs with $\mathrm{T}$ spectral type with the aim of shedding light on the problem of the form of the stellar mass function at the lower end, an issue that has been identified as a key VO-Science case both by AstroGrid and EURO-VO. A similar methodology will be apply for the exploitation of UKIDSS, a near-IR survey three magnitudes deeper than 2MASS and whose first Data Release took place in July 2006. With UKIDSS it will be possible to discover faint T-type dwarfs beyond the 2MASS limiting magnitude and to proof the existence of the theoretically predicted Y-type brown dwarfs, a class of objects cooler than the T-type dwarfs and whose discovery constitutes one of the key science drivers for the project.

- Characterization of star forming regions

Taking advantage of VO tools we have built the mass function of the $\sigma$ Orionis cluster from 20 to $1 \mathrm{M}_{\odot}$. Membership criteria based on proper motions and colour-magnitude diagrams have permitted the identification of four new cluster members. A study of the $1-0.05 \mathrm{M}_{\odot}$ regime, down to the brown dwarf domain, by cross-matching 2MASS and DENIS is presently ongoing. In the short-term our aim is to repeat the same type of analysis for all the clusters of the Orion OB1b association.

- Formation of brown dwarfs

The way how brown dwarfs are formed is still a matter of debate. Among the competing theories, Reipurth \& Clarke (2001, AJ, 122, 432) proposed that brown dwarfs are stellar embryos ejected from the formation region due to gravitational interactions before their hydrostatic cores could build up enough mass to eventually start hydrogen burning.

Our goal is to check this model by cross-correlating IPHAS (Drew et al. 2005, MNRAS, $362,753)$ and 2MASS to search, for the first time, young brown dwarfs over large areas of the sky on the basis of their $\mathrm{H} \alpha$ emission and IR colors. Preliminary analysis of the follow-up observations of some of the candidates seems to confirm the existence of, at least, one young brown dwarf among them. 